\title{
Fabricação digital na América do Sul: um mapeamento de linhas de ação a partir da arquitetura e urbanismo
}

\author{
Digital Fabrication in South America: mapping lines of action from architecture and urbanism \\ David M. Sperling \\ Universidade de São Paulo, Brasil \\ sperling@sc.usp.br \\ - Pablo C. Herrera \\ Universidad Peruana de Ciencias Aplicadas, Peru \\ Pablo@espaciosdigitales.org
}

\begin{abstract}
The article presents a mapping of digital fabrication laboratories in South America from the architecture and urbanism field. First, it draws a brief context of implementation of facilities and growing of expertise highlighting economic, academic and cultural aspects. Second, it presents some data mapped from 31 laboratories of the region, as infrastructure, and correlations between uses and applications. Third, it organizes the mapped laboratories in two significant approaches for the region's context: works focused on technological development and actions directed to the social and environmental development. Fourth, it infers some possible steps of the field in the region in the near future.
\end{abstract}

Keywords: Digital Fabrication, Laboratories, South America, Technological Development, Social and Environmental Development

\section{Introdução}

O artigo apresenta um mapeamento de laboratórios de fabricação digital orientados para a arquitetura e o urbanismo na América do Sul, organizando-os a partir de duas linhas de ação significativas para o contexto da região: a atuação focada em desenvolvimento tecnológico e a atuação direcionada ao desenvolvimento social e ambiental.

Breve contexto de implementação de laboratórios de fabricação digital na região

Do surgimento das primeiras instalações no início dos anos 2000 ao atual estágio de expansão por meio de instituições de ensino e pesquisa e de iniciativas independentes, o processo de implementação de laboratórios de fabricação digital na América do Sul pode ser caracterizado primordialmente segundo três aspectos principais: econômicos, acadêmicos e culturais. Cada um destes aspectos, por sua vez, pode ser caracterizado por três estágios subsequentes e coexistentes, ou seja, a condição do aparecimento de um estágio não elimina o(s) anterior(es), mas o(s) reposiciona.

Em termos econômicos, em um primeiro estágio marcado pelo alto custo dos equipamentos de prototipagem rápida, as principais iniciativas se vincularam à indústria pesada, às universidades e instituições de pesquisa com capital para investimento. Em seguida, com a redução dos custos para aquisição de equipamentos e a expiração de algumas patentes
(Herrera, Juarez, 2013), passaram a emergir laboratórios menores, focados em design de produto, equipados com máquinas de menor porte destinadas a pequenas indústrias. Em anos recentes, a expansão ocorre de forma significativa na escala do usuário doméstico, a partir da maior oferta de máquinas produzidas localmente, do menor preço e da popularização de equipamentos de manufatura aditiva notadamente as impressoras 3D.

Em termos acadêmicos, pode-se delinear um primeiro momento de organização de laboratórios de fabricação digital na área de arquitetura decorrente de intercâmbios e pesquisas de professores no exterior - tendo os Estados Unidos, a Inglaterra e a Espanha como destinos principais (Sperling, Herrera e Scheeren, 2015). Par-e-passo surgiram laboratórios constiuídos pela iniciativa institucional ou pessoal de professores da área, sem a decorrência direta de intercâmbios em fabricação digital com o exterior, mas a partir de referências regionais e internacionais, seja por contatos (inter)nacionais de pesquisa ou pela participação em eventos como o Sigradi e as sociedades irmãs. $O$ momento atual pode ser caracterizado pelo aparecimento de nucleações internas à região, a partir da formação de pesquisadores, professores e arquitetos "nativos (fabricantes) digitais" que passam a constituir laboratórios em instituições de ensino e pesquisa, ou escritórios direcionados às tecnologias digitais de fabricação.

Em termos culturais, pode-se indicar um primeiro momento composto por iniciativas com o objetivo de 
introduzir e difundir o campo da fabricação digital na região a partir de práticas e produções referenciadas em grandes centros na área - constituindo um movimento de conexão entre o local e o global. Em seguida, passam a surgir iniciativas interessadas em ampliar a diversidade de direcionamentos até então conferidos à fabricação digital a partir de condicionantes locais - conformando um movimento de amplificação de interesse pelas especificidades do local. Atualmente, com o crescimento do movimento maker, vem ocorrendo a ampliação exponencial de plataformas compartilhadas de pequena, média e grande escala para fabricação digital - conformando um movimento de conexão em rede entre makers e máquinas. Um indicativo importante desta situação que se apresenta é o cadastro atual de 762 hubs de impressão digital na América Latina no portal 3D Hubs, de um total de 21.908 no mundo - dentre os países da região com mais cadastros, Brasil: 240; Colômbia: 60; Argentina: 51; Chile: 30 (dados de 11/09/2015). Neste contexto, é possível afirmar não ser relevante a especialização de uma determinada plataforma de fabricação, mas a sua disponibilidade para acesso em rede.

É possível ainda considerar como significativas as correlações entre cada estágio dos aspectos econômico, acadêmico e cultural do processo de implementação de laboratórios de fabricação digital na América do Sul, como mostra a Tabela 1.

Tabela 1: Estágios de implementação de laboratórios de fabricação digital na América do Sul

\begin{tabular}{|l|l|l|l|}
\cline { 2 - 4 } \multicolumn{1}{c|}{} & $\begin{array}{l}\text { Aspecto } \\
\text { Econômico }\end{array}$ & $\begin{array}{l}\text { Aspecto } \\
\text { Acadêmico }\end{array}$ & $\begin{array}{l}\text { Aspecto } \\
\text { Cultural }\end{array}$ \\
\hline $\begin{array}{l}\text { Primeiro } \\
\text { Estágio }\end{array}$ & $\begin{array}{l}\text { Alto custo } \\
\text { de máquinas } \\
\text { concentradas } \\
\text { na indústria e } \\
\text { em instituições } \\
\text { de ensino e } \\
\text { pesquisa }\end{array}$ & $\begin{array}{l}\text { Pesquisa e } \\
\text { intercâmbios } \\
\text { no exterior }\end{array}$ & $\begin{array}{l}\text { Conexões } \\
\text { local-global }\end{array}$ \\
\hline $\begin{array}{l}\text { Segundo } \\
\text { Estágio }\end{array}$ & $\begin{array}{l}\text { Redução } \\
\text { de custo de } \\
\text { máquinas de } \\
\text { médio porte } \\
\text { Quebra de } \\
\text { patentes }\end{array}$ & $\begin{array}{l}\text { Referenciais } \\
\text { regionais e in- } \\
\text { ternacionais } \\
\text { Contatos } \\
\text { (inter) } \\
\text { nacionais de } \\
\text { pesquisa }\end{array}$ & $\begin{array}{l}\text { Ampliação } \\
\text { de interesse } \\
\text { pelas } \\
\text { especifida- } \\
\text { des locais }\end{array}$ \\
\hline $\begin{array}{l}\text { Terceiro } \\
\text { Estágio }\end{array}$ & $\begin{array}{l}\text { Redução geral } \\
\text { de custo de } \\
\text { máquinas } \\
\text { Máquinas } \\
\text { produzidas na } \\
\text { região } \\
\text { Acesso } \\
\text { doméstico }\end{array}$ & $\begin{array}{l}\text { Nucleação } \\
\text { interna } \\
\text { "Nativos } \\
\text { (fabricantes) } \\
\text { digitais" }\end{array}$ & $\begin{array}{l}\text { Conexões em } \\
\text { rede entre } \\
\text { makers e } \\
\text { máquinas }\end{array}$ \\
\hline
\end{tabular}

Ao longo da última década, o número de iniciativas de implementação de laboratórios, contratação de serviços, organização de workshops e proposição de disciplinas acadêmicas têm aumentado exponencialmente na área de arquitetura e urbanismo na região, exigindo a necessidade de maior compreensão sobre este processo em andamento.

\section{Metodologia}

Este artigo explora temas apresentados por Celani (2014), o levantamento realizado para a organização da Exposição "Homo Faber: Digital Fabrication in Latin America" (São Paulo, 2015) e dados complementares aos utilizados em artigo recentemente publicado (Sperling, Herrera e Scheeren, 2015).

Com o objetivo de mapear o estado da arte da fabricação digital na região, um formulário online foi elaborado e enviado a 48 dos 70 laboratórios estimados na região orientados à área de arquitetura (vinculados ou não à rede MIT Fab e Rhino Fab). Destes, 31 laboratórios enviaram respostas consistentes, sendo eles dos seguintes países: Argentina (2), Brasil (22), Colômbia (1), Chile (4), Peru (1) e Uruguai (1) - (Figura 1).

Os laboratórios participantes foram: Argentina: CID - Centro de Informática y Diseño - FADU/Universidad Nacional del Litoral; Instituto de la Espacialidad Humana Laboratorio de Morfología - FADU/UBA; Brasil: Aleph Zero; CADEP - Centro Avançado de Desenvolvimento de Produtos - FAAC/UNESP; Centro de prototipagem experimental FCT/UNESP; DT3D - Divisão de Tecnologias Tridimensionais Centro de Tecnologia da Informação Renato Archer; Estudio Guto Requena; Fab Lab Universidade de São Paulo - FAU/ USP; Fab Social; Garagem Fab Lab; GEGRADI, Grupo de Estudos para o Ensino/aprendizagem de Gráfica Digital - FAU - UFPel; LAGEAR - Laboratório Gráfico para Experimentação Arquitetônica - FAU/UFMG; Lamo3D - Laboratório de modelos 3D e Fabricação Digital - FAU/UFRJ; LAPAC Laboratório de Automação e Prototipagem para a Arquitetura e Construção - FEC/UNICAMP; Laboratório de Prototipagem Rápida Mackenzie - FAU/Mackenzie; LEAUD - Laboratório de Estudo das Linguagens e Expressões da Arquitetura, Urbanismo e Design - DAUR/UFJF; LED - Laboratório de Experiência Digital - FAU/UFC; LM+P - Laboratório de Modelos e Prototipagem - DA-CT/UFPA; Nomads - IAU/ USP; PRONTO 3D - Laboratório de Prototipagem e Novas Tecnologias Orientadas ao 3D - Design/UFSC; Rede Brasileira de Fabricação Digital; SimmLab - Laboratório de Simulações e Modelamento em Arquitetura e Urbanismo - FAU/UFRGS; SUBdV Architecture; ViD_Virtual Design - Design/UFRGS; Chile: Area Computacional - Universidad Tecnica Federico Santa Maria; gt2P - Great things to people; Lab CNC FAU/ Uchile; Producción Digital UC / Fabhaus UC - PUC-Chile; Colômbia: Frontis3D R+D; Peru: Fab Lab Lima; Uruguai: LabFabMVD - FArq/Universidad de la República.

O formulário teve o objetivo de captar informações institucionais (nome, filiação institucional, coordenador atual, equipe atual, data de fundação, breve histórico, associação ou não a redes, endereço físico/e-mail de contato/website) e infraestrutura (tecnologias de fabricação, número/tipos/ marcas de equipamentos), atividades (workshops e cursos), 
usos e aplicações da fabricação digital. Os itens "usos" e "aplicações" dizem respeito, respectivamente, à característica intrínseca do objeto fabricado e ao campo a que se destina - por exemplo, o uso da fabricação digital para a produção de modelos arquitetônicos a serem aplicados como modelos para ensino.

As possibilidades de respostas múltiplas para "usos de fabricação digital" foram assim definidas: "fabricação de modelos arquitetônicos"; "fabricação de moldes para construção"; "fabricação de máquinas"; "prototipagem de pequenos objetos"; e "outros". E para "aplicações dos processos de fabricação e objetos fabricados" as possibilidades múltipla escolha foram assim definidas: "protótipos de design (visualização e/ou simulação e/ou análise)"; "modelos para ensino"; "componentes para a indústria da construção"; "modelos para arte e museologia"; "modelos de edifícios históricos"; "objetos para pessoas com necessidades especiais"; "objetos/processos para o desenvolvimento de comunidades"; e "outros".

\section{Resultados}

O confronto entre as informações institucionais, infraestrutura, atividades, usos e aplicações deste conjunto significativo de 31 laboratórios permitiu o delineamento de um quadro da fabricação digital na região, sistematizado por meio de um conjunto de diagramas, alguns apresentados aqui.

Como mostra a Figura 2, dos 31 laboratórios mapeados,
22 estão concentrados em universidades e instituições de pesquisa acadêmica e 9 são escritórios privados. Da totalidade, 29 laboratórios possuem pelo menos uma impressora 3D, 23 possuem pelo menos uma cortadora a laser, 21 possuem pelo menos uma router CNC, 14 possuem outro tipo de equipamento de fabricação e 11 terceirizam serviços para os quais não possuem máquinas.

Entre os laboratórios de instituições de pesquisa acadêmica, 13 possuem grande variedade de equipamentos, 6 possuem uma variedade média e 3 possuem pequena variedade. Entre os laboratórios privados, 3 estão vinculados às redes MIT Fab e Rhino Fab e, portanto, possuem uma variedade média de equipamentos; e 3 possuem pequena variedade ou terceirizam a totalidade de seus trabalhos. Este comparativo mostra uma diferença significativa de infraestrutura existente entre os dois grupos, sinalizando que os grandes investimentos em infraestrutura de laboratórios de fabricação digital na área de arquitetura estão localizados nas instituições de pesquisa.

A Figura 3 sistematiza a atuação dos laboratórios em função de workshops e cursos que ministram, além de usos e aplicações que fazem da fabricação digital. É notório que a totalidade afirmou estar de alguma forma vinculada a atividades de formação (workshops, cursos e pesquisa). Enquanto os "usos" indicam uma predominância de pequenos modelos, protótipos e objetos, seguidos de forma equitativa pelas outras categorias, as "aplicações" indicam o ensino como o foco principal.

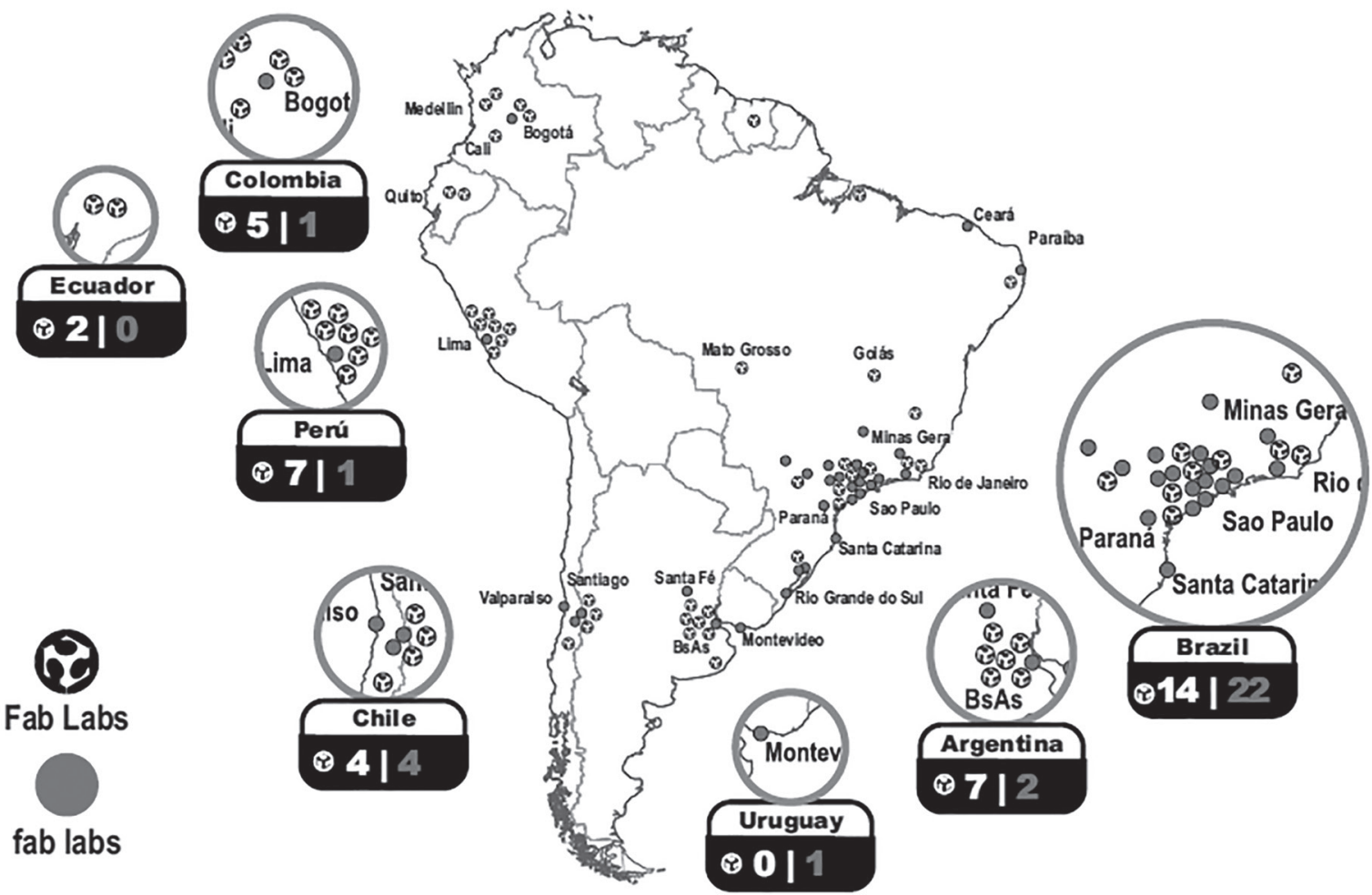

Fifura 1. 


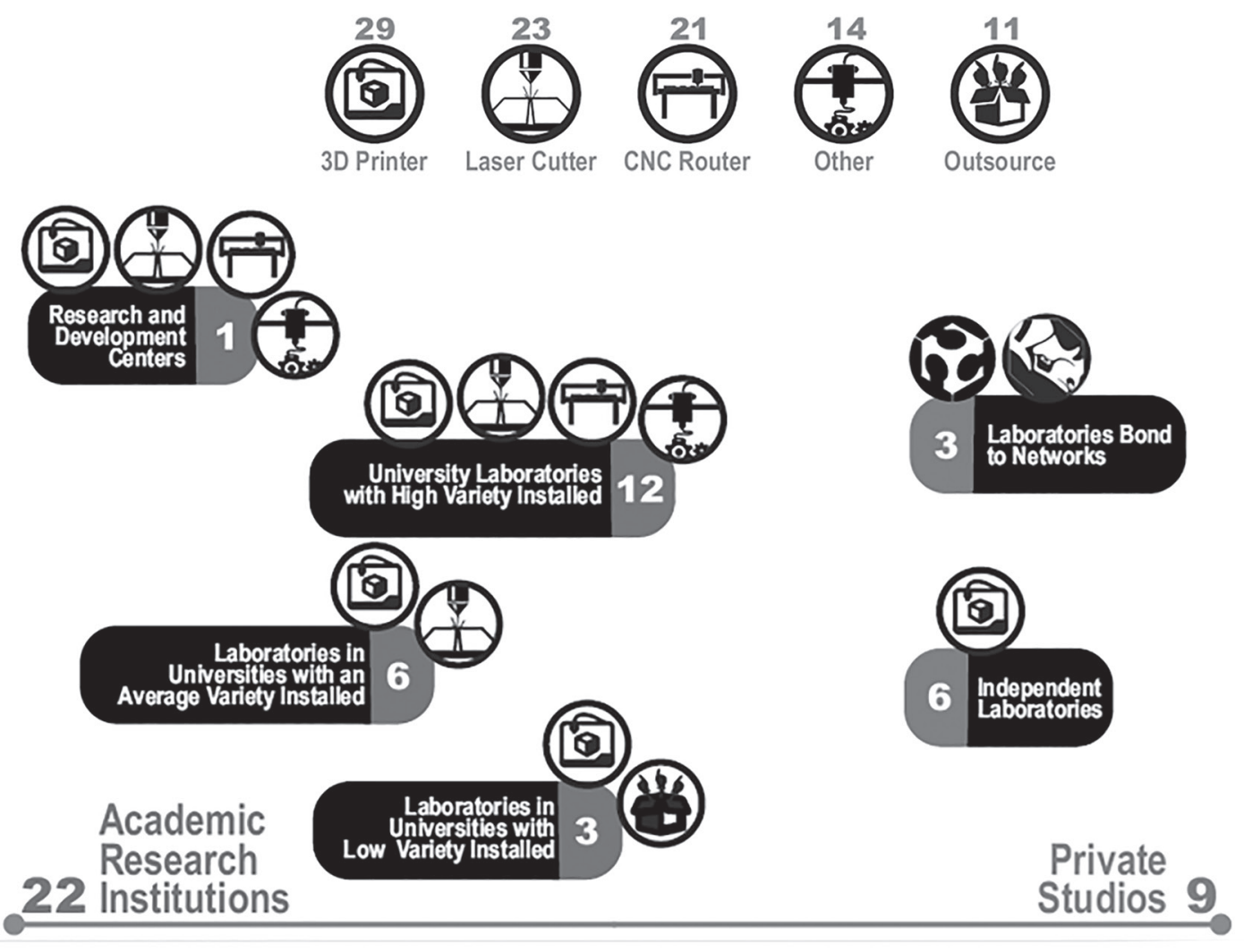

Fifura 2.
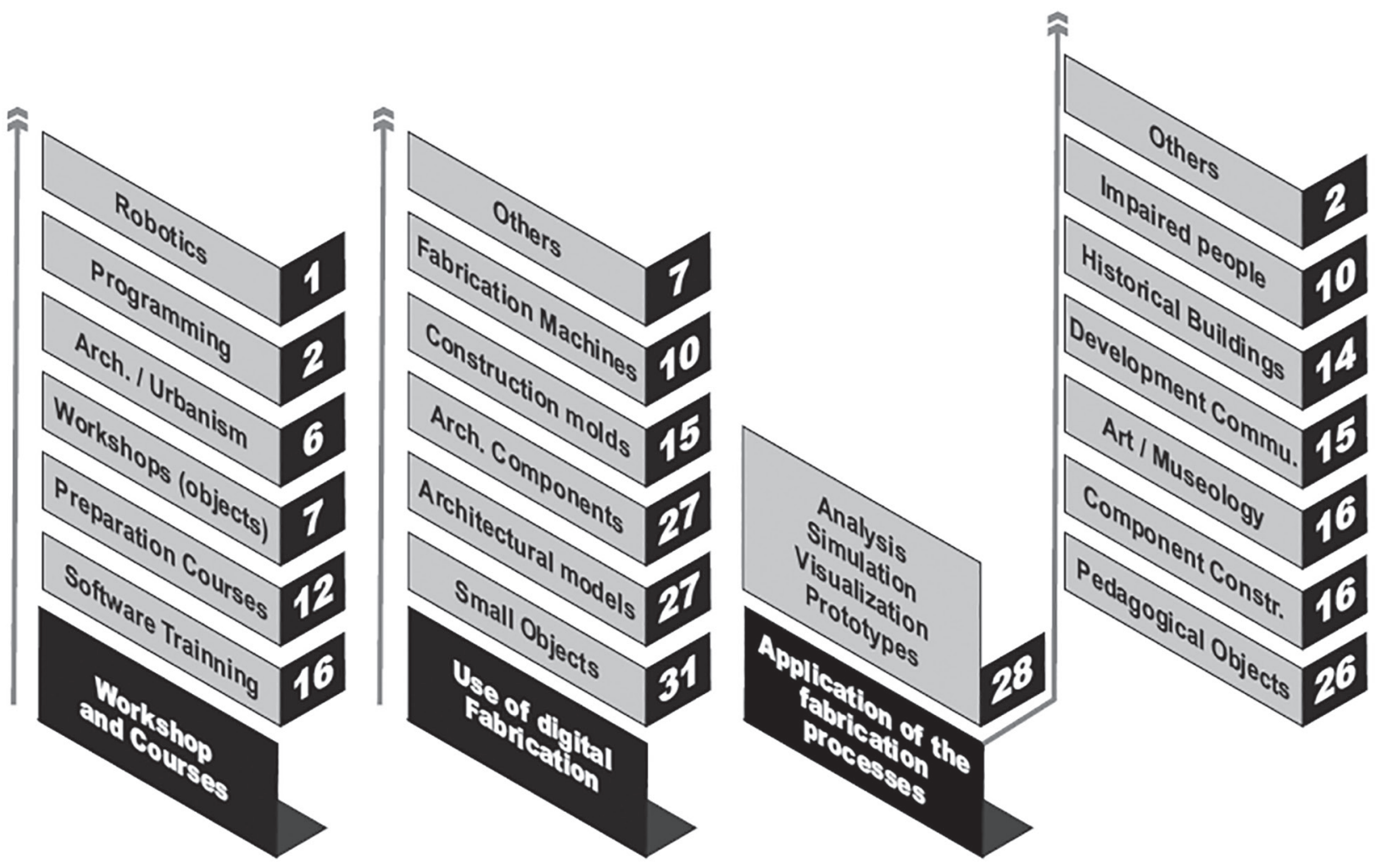

Fifura 3. 
Linhas de ação: desenvolvimento tecnológico e desenvolvimento social e ambiental

Correlações entre "usos" e "aplicações" apontados pelos laboratórios mapeados indicam dois direcionamentos principais de atuação: desenvolvimento tecnológico e desenvolvimento social e ambiental. É certo que a fabricação digital tem em termos gerais um claro acento de desenvolvimento tecnológico a partir da introdução de novos conhecimentos e processos direcionados à materialização. $\mathrm{O}$ que o mapeamento permite apontar é a indicação espontânea por parte de $48 \%$ dos laboratórios da investigação de "usos" e "aplicações" voltados para ações de desenvolvimento social e ou ambiental por meio da fabricação digital

Indicaram a linhas de ação voltadas primordialmente ao desenvolvimento tecnológico da arquitetura os seguintes laboratórios: Argentina: CID - Centro de Informática y Diseño - FADU/Universidad Nacional del Litoral; Brasil: CADEP - Centro Avançado de Desenvolvimento de Produtos - FAAC/UNESP; Centro de prototipagem experimental - FCT/ UNESP; DT3D - Divisão de Tecnologias Tridimensionais Centro de Tecnologia da Informação Renato Archer; Laboratório de Prototipagem Rápida Mackenzie - FAU/ Mackenzie; LEAUD - Laboratório de Estudo das Linguagens e Expressões da Arquitetura, Urbanismo e Design - DAUR/ UFJF; LM+P - Laboratório de Modelos e Prototipagem - DACT/UFPA; PRONTO 3D - Laboratório de Prototipagem e Novas Tecnologias Orientadas ao 3D - Design/UFSC; Rede Brasileira de Fabricação Digital ; SimmLab - Laboratório de Simulações e Modelamento em Arquitetura e Urbanismo - FAU/ UFRGS; ViD_Virtual Design - Design/UFRGS; Chile: Area Computacional - Universidad Tecnica Federico Santa Maria; Lab CNC FAU/Uchile; Producción Digital UC / Fabhaus UC PUC-Chile; Colômbia: Frontis3D R+D; Uruguai: LabFabMVD FArq/Universidad de la República.

Indicaram espontaneamente linhas de ação que pretendem incorporar, ao desenvolvimento tecnológico da arquitetura, ações de desenvolvimento social e ambiental os seguintes laboratórios: Argentina: Instituto de la Espacialidad Humana - Laboratorio de Morfología - FADU/UBA; Brasil: Aleph Zero; Estudio Guto Requena; Fab Lab Universidade de São Paulo - FAU/USP; Fab Social; Garagem Fab Lab; GEGRADI, Grupo de Estudos para o Ensino/aprendizagem de Gráfica Digital - FAU - UFPel; LAGEAR - Laboratório Gráfico para Experimentação Arquitetônica - FAU/UFMG; LAPAC - Laboratório de Automação e Prototipagem para a Arquitetura e Construção - FEC/UNICAMP; Lamo3D Laboratório de modelos 3D e Fabricação Digital - FAU/UFRJ; LED - Laboratório de Experiência Digital - FAU/UFC; Nomads - IAU/USP; SUBdV Architecture; Chile: gt2P - Great things to people; Peru: Fab Lab Lima.

Na categoria "usos", enquanto a "fabricação de moldes para a construção" e "fabricação de componentes arquitetônicos" receberam direcionamento maior para o desenvolvimento tecnológico, os outros itens (fabricação de modelos arquitetônicos; fabricação de máquinas; prototipagem de pequenos objetos; e outros) tiveram direcionamento tanto para o desenvolvimento tecnológico, quanto social e ambiental.

$\mathrm{Na}$ categoria "Aplicações", enquanto "protótipos de design (visualização e/ou simulação e/ou análise)", "modelos para ensino" e "modelos para arte e museologia" indicaram direcionamento duplo, "componentes para a indústria da construção" e "invenção de máquinas" foram direcionados para o desenvolvimento tecnológico e "modelos de edifícios históricos", "objetos para pessoas com necessidades especiais" e "objetos/processos para o desenvolvimento de comunidades" foram direcionados para o desenvolvimento social e ambiental.

Por fim, as linhas de ação direcionadas ao desenvolvimento tecnológico e ao desenvolvimento social e ambiental, significativas para o contexto da América Latina, estão presentes tanto em laboratórios vinculados a universidades como na atuação de laboratórios independentes. A figura 4 sintetiza o mapeamento das informações quanto às linhas de ação.

\section{Linhas de ação: possíveis futuros próximos, equipamentos e materiais}

É importante esclarecer que esse movimento na América do Sul seguiu sempre atrás de fenômenos semelhantes ocorridos na Europa e Estados Unidos, onde os primeiros laboratórios voltados para a área de arquitetura e urbanismo surgiram no final dos anos 1990.

Por um lado, esta antecedência permite prever que a próxima etapa na evolução dos laboratórios latinoamericanos será - seguindo a mesma lógica - a incorporação dos braços robóticos (como Kuka ou $\mathrm{ABB}$ ), já presentes em algumas escolas de arquitetura europeias e americanas, e na região já comparecem na Universidad Adolfo Ibañez e Universidad Teçnica Frederico Santa Maria (Chile). E, no Brasil, há experimentos de desenvolvimento de pequenos braços robóticos, como na Universidade de São Paulo e na Universidade Federal de Santa Catarina. A tendência é que eles passem inclusive a substituir máquinas como as fresadoras CNC, tendo em vista sua maior flexibilidade e a tendência de queda dos preços. Outra conclusão que se pode extrair é que até agora predominaram os equipamentos de fabricação aditiva (impressoras 3D) e subtrativa (cortadoras a laser, plotadoras de recorte e fresadoras $\mathrm{CNC}$ ). Um tipo de equipamento que ainda não se encontra disponível em tamanho e versão compatíveis com laboratórios de ensino são os equipamentos de conformação, mas os braços robóticos podem ser utilizados para esta finalidade, em especial para a dobragem de chapas metálicas, tal como já está ocorrendo (Lavallee, Vroman, Keshet, 2011)

Por outro lado, seguindo a dinâmica econômica regional, este processo será mais lento, considerando-se, por exemplo, o intervalo de 10 anos entre as poucas ações no presente e a utilização por Gramazio na ETH Zurique (conforme a palestra "The Informed Wall: Applying Additive Digital Fabrication Techniques on Architecture", na ACADIA 2006). Isto se deve 


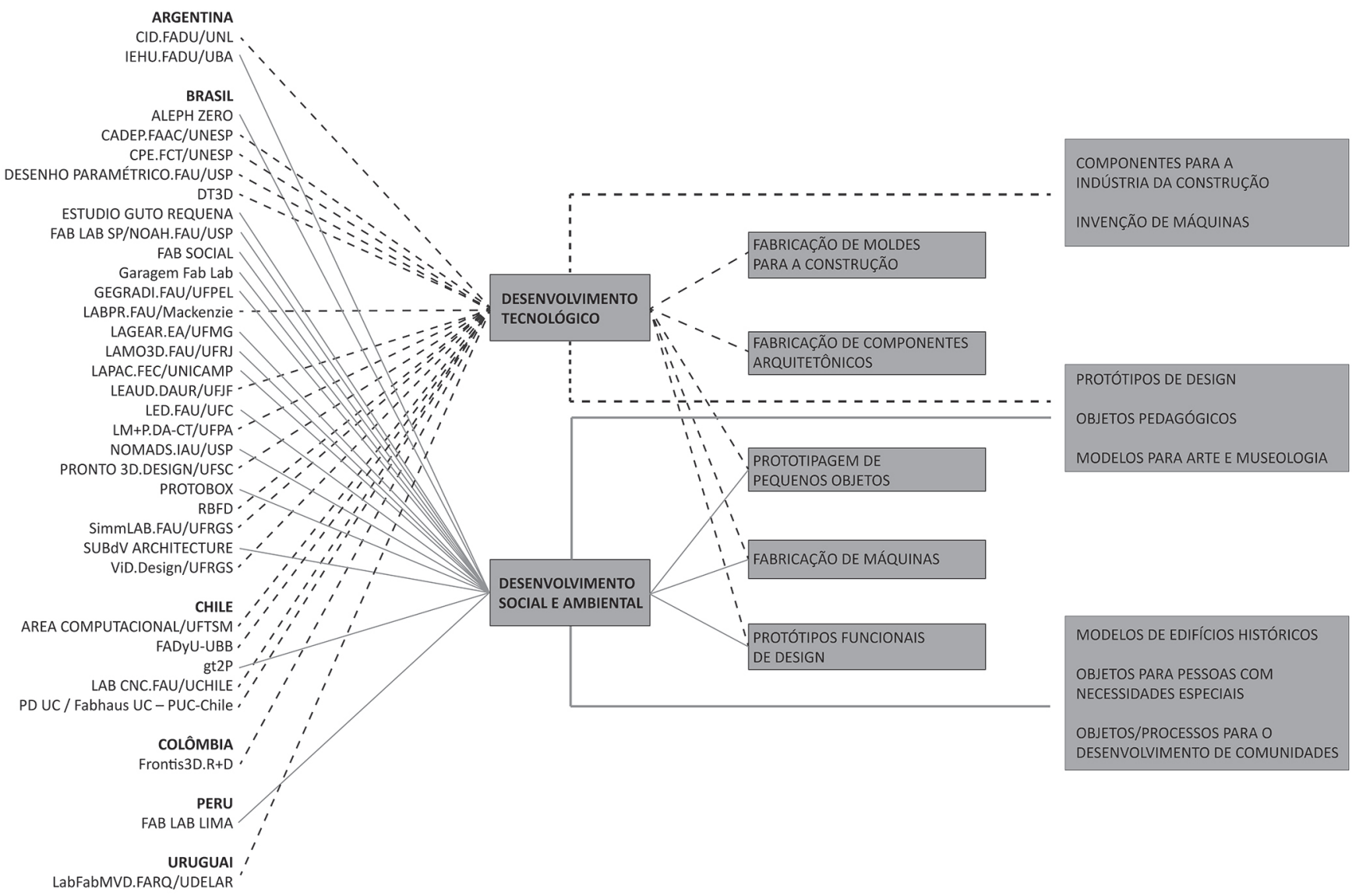

Figura 4: Correlações entre linhas de ação, usos e aplicações da fabricação digital pelos laboratórios mapeados.

ao alto custo de implementação, manutenção e treinamento.

Seguindo esta dinâmica, o movimento parece se direcionar preferencialmente à ampliação da capacidade instalada ao invés da aquisição de novas tecnologias, como os braços robóticos. Seé certo que os estudantes de mestrado e doutorado que retornam com o conhecimento adquirido em instituições do exterior têm condições de aplicá-lo, o alto investimento em tecnologias ainda incipientes na região indica que as possibilidades de sua aplicação não se darão de imediato.

Em relação ao avanço das pesquisas e aplicações de braços robóticos em fabricação digital orientada para a arquitetura, o cenário mostra-se muito embrionário. O Brasil - como um dos representantes deste avanço - inicia uma fase econômica que indica uma limitação de recursos para pesquisa neste setor. $O$ Chile vem desenvolvendo sua aplicação como uma diferença comercial, a partir de duas universidades particulares que já compraram estes equipamentos e são as únicas que poderiam fazê-lo. Nos outros países da região com maior experiência na área (Argentina, Colômbia, Peru, Uruguai) não há ações nesta direção, enquanto países que iniciam na fabricação digital (Bolívia e Equador) o fazem com equipamentos básicos.

Esta dinâmica regional parece abrir, portanto à ampliação do uso das impressoras 3D (o equipamento com maior comparecimento em nossa pesquisa - 29 em 31 laboratórios). $\mathrm{O}$ uso de equipamentos como Makerbot, por exemplo, que requerem somente a substituição do cabeçote extrusor com menos de $7 \%$ do custo do equipamento, permitirá outros materiais além dos plásticos, como filamentos compostos de madeira, pedra, bronze e metais.

$\mathrm{Na}$ direção da investigação acerca de tecnologias de manufatura aditiva com uso de novos materiais, Silveira et all (2014) desenvolveram no Brasil, a partir da máquina Fab@ Home, a Fab@CTI 3-D Printer. O experimento consistiu na concepção e teste de um cabeçote intercambiável alimentado por materiais sintéticos reutilizados ou biomateriais, ambos em pó, os quais são fundidos e aplicados continuamente.

\section{Discussão}

O mapeamento mostra que há "clusters" de laboratórios concentrados principalmente na região sudeste do Brasil, no centro da Argentina, na região metropolitana de Santiago (Chile), Lima (Perú) e Bogotá (Colômbia), indicando haver um movimento de concentração de laboratórios em capitais e centros regionais.

Embora seja possível delinear um terceiro estágio de 
implementação de laboratórios na região que articula um maior acessibilidade a máquinas, a nucleação regional recente de novos laboratórios em universidades e escritórios privados a partir da formação de "nativos (fabricantes) digitais" e a crescente participação em hubs que conectam makers e máquinas, as ações na região mostram-se fortemente capitaneadas pelos laboratórios de universidades de maior porte, destacando-se, no Brasil, a Universidade de São Paulo; a Universidade Estadual de Campinas; a Universidade Federal de Minas Gerais; a Universidade Federal do Rio de Janeiro; e a Universidade Federal de Santa Catarina; na Argentina, a Universidad de Buenos Aires; no Chile, a Universidad de Chile, no Peru, a Universidad Peruana de Ciencias Aplicadas; e, no Uruguai, a Universidad de la República.

Espera-se em estudo futuro mapear os efeitos que ainda não são claros no campo da arquitetura do que chamamos aqui de "terceiro estágio" de implementação de laboratórios de fabricação digital na região. Por ora, é possível perceber uma complementaridade entre a capacidade de investimento em infraestrutura e atualização constante de equipes de pesquisa pelos laboratórios de universidades e centros de pesquisa, e a capacidade de prospecção de segmentos promissores de atuação e aplicação de tecnologias pelas iniciativas independentes recentes (escritórios e organismos privados).

Tanto uns quanto outros estão presentes nas duas linhas de ação que identificamos como primordiais, sendo que quase metade dos laboratórios mapeados indica espontaneamente investigarem articulações do desenvolvimento tecnológico com aspectos sociais e ambientais por meio da fabricação digital.

Em relação às linhas de ação referentes a novas tecnologias, estima-se que a pesquisa com materiais em manufatura aditiva se apresentará como perspectiva em curto prazo, a ser seguida, a médio prazo, pela compra de novos equipamentos como braços robóticos.

\section{Referências}

Celani, G. (2014). Espaços para a interdisciplinaridade: laboratórios de fabricação digital na pesquisa, ensino e extensão. In: Arlindo Philippi Jr; Valdir Fernandes. (Org.). Práticas da interdisciplinaridade no ensino e pesquisa. 1ed. São Paulo: Manole, v. 1, p. 747-764.

Herrera, P. C.; Juarez, B. (2013). Fabrication Laboratories: Problems and possibilities of implementation in Latin America. In: Proceedings of the Fab 9 Research Stream. Yokohama, Keiko University SFC. URL: http://www.fablabinternational. org/fab-lab-research/proceedings-from-the-fab-9-researchstream

Lavallee, J.; Vroman, R.; Keshet, Y. (2011) Automated Folding of Sheet Metal Components with a Six-axis Industrial Robot, ACADIA 11: Integration through Computation [Proceedings of the 31st Annual Conference of the Association for Computer Aided Design in Architecture (ACADIA)] Banff (Alberta) 13-16 October, 2011, pp. 144-151

Sperling, D. M. ; Herrera, P. C. (2015). Homo faber: digital fabrication in latin america CAAD futures 2015> the next city. 1. ed. São Carlos: Instituto de Arquitetura e Urbanismo.

Sperling, D. M. ; Herrera, P. C. ; Scheeren, R. Migratory Movements of Homo Faber: Mapping Fab Labs in Latin America. Communications in Computer and Information Science. 1ed.: Springer Berlin Heidelberg, 2015, p. 405-421.

Silveira, Z.; Freitas, M.; Neto, P.; Noritomi, P.; Silva, J. (2014) Design development and functional validation of an interchangeable head based on mini screw extrusion applied in an experimental desktop 3-D printer. International Journal of Rapid Manufacturing (Print), v. 4, p. 49-65.

Sperling, D. M. ; Herrera, P. C. ; Scheeren, R. (2015). Migratory Movements of Homo Faber: Mapping Fab Labs in Latin America. Communications in Computer and Information Science. 1ed.: Springer Berlin Heidelberg, 2015, p. 405-421. 